DOI: $10.22481 /$ recuesb.v8i14.7849

\title{
PROJETO DE EXTENSÃO VIDA ATIVA - UNATI: RELATO DE EXPERIÊNCIA DURANTE A PANDEMIA PELA COVID-19
}

\section{EXTENSION PROJECT ACTIVE LIFE - UNATI: EXPERIENCE REPORT DURING THE COVID-19 PANDEMIC}

\author{
Fernanda Cardoso Silva ${ }^{1}$ \\ Ítalo Gabriel Ferreira Germano ${ }^{2}$ \\ Nívea Akemi Ando Teixeira ${ }^{3}$ \\ Karla Caroline Oliveira ${ }^{4}$ \\ Rafaela de Oliveira Martins ${ }^{5}$ \\ Carolina Kosour ${ }^{6}$
}

\begin{abstract}
Resumo: Introdução: Devido à pandemia pela COVID-19, houve a necessidade de adaptação nas atividades de promoção à saúde, assim, o Projeto de Extensão Vida Ativa, diante da impossibilidade de sua aplicação presencial, realizou adaptações para ser desenvolvido de forma remota. Objetivo: Relatar as ações desenvolvidas pelos alunos e professores do Projeto Vida Ativa para pessoas idosas durante o período de isolamento social ocasionado pelo Covid19. Metodologia: Trata-se de relato de experiência das ações desenvolvidas pelo Projeto de Extensão Vida Ativa durante a pandemia pela COVID-19. Foram realizadas atividades de forma remota pelas redes sociais WhatsApp ${ }^{\circledR}$ e Facebook®. Resultados: Os idosos participantes do projeto relataram melhorias propiciadas pelas ações e como elas influenciaram positivamente a qualidade de vida dos mesmos durante o isolamento social. Os acadêmicos participantes do projeto, alcançaram maior envolvimento com os idosos participantes, proporcionando interação dialógica em formato digital. São realizadas pelos alunos
\end{abstract}

\footnotetext{
1 Graduanda em Fisioterapia, Universidade Federal de Alfenas, Alfenas, Minas Gerais, Brasil. E-mail: fernanda.cardoso@sou.unifal-mg.edu.br

2 Graduando em Fisioterapia, Universidade Federal de Alfenas, Alfenas, Minas Gerais, Brasil. E-mail: italo.germano@sou.unifal-mg.edu.br

3 Graduanda em Fisioterapia, Universidade Federal de Alfenas, Alfenas, Minas Gerais, Brasil. E-mail: nivea.teixeira@sou.unifal-mg.edu.br

4 Graduanda em Fisioterapia, Universidade Federal de Alfenas, Alfenas, Minas Gerais, Brasil. E-mail: karla.oliveira@sou.unifal-mg.edu.br

5 Graduanda em Fisioterapia, Universidade Federal de Alfenas, Alfenas, Minas Gerais, Brasil. E-mail: rafaela.martins@sou.unifal-mg.edu.br

${ }^{6}$ Professora Doutora em Ciências Cirúrgicas, professora da Universidade Federal de Alfenas, Alfenas, Minas Gerais, Brasil. Orcid: https://orcid.org/0000-0001-6479-9550 E-mail: carolina.kosour@unifal-mg.edu.br
} 
apresentações dos resultados de pesquisas de forma online em eventos científicos. Conclusão: As atividades desenvolvidas de forma remota no projeto proporcionaram repercussão positiva na manutenção funcional e melhora da qualidade de vida dos idosos e impacto na formação do aluno promovendo os princípios da extensão: indissociabilidade, interdisciplinaridade, interação dialógica, impacto na formação do aluno e social. Portanto, ações de extensão em saúde de forma remota devem ser estimuladas, pois promovem a aproximação da comunidade com o conhecimento acadêmico.

Palavras-chave: Fisioterapia. Saúde do Idoso. Pandemia. Isolamento Social. Prevenção.

Abstract: Due to the COVID-19 pandemic, there was a need for adaptation of the activities of health promotion. In light of the impossibility of presential application, Extension Project Active Life made some adaptations to be done at a remote way. Aim: Report activities developed by Active Life's Project students and professors, aimed at elderly people during the covid-19 social distancing. Methodology: This is an experience report of the actions developed by Extension Project Active Life during COVID-19 pandemic. Activities were carried out remotely through the social networks WhatsApp ${ }^{\circledR} \square$ and Facebook ${ }^{\circledR} \square$. Results: The elderly participants in the project reported improvements provided by the project's actions and how they positively influenced their quality of life during social isolation. The academics that participate at the project, achieved greater involvement with the elderly participants by providing dialogical interaction in digital format. The students also presented research results in online scientific events. Conclusion: the activities developed remotely at the project, provided a positive repercussion of the functional maintenance and the improving of quality life in elderly and positive impact on the student academic training, promoting the principles of extension: inseparability, interdisciplinarity, interaction between different points of view, social impact and impacts in the student's academic formation.

Keywords: Physiotherapy. Social Isolation. Pandemic. Prevention. Health of the Elderly

\section{Introdução}

No Brasil, de acordo com projeções do Instituto Brasileiro de Geografia e Estatística (IBGE), até 2060, a população idosa corresponderá a aproximadamente $25 \%$ da população. Isso ocorre principalmente devido ao processo de transição epidemiológica em que houve aumento de doenças crônicas, reforçando a importância de ações multidisciplinares para manutenção da saúde desses indivíduos (CORTEZ et al., 2019). 
Com o envelhecimento ocorrem alterações fisiológicas sistêmicas como a redução do equilíbrio, diminuição da força muscular e rigidez articular. Também se pode observar redução da complacência e expansibilidade pulmonar, redução da força dos músculos respiratórios (PASCOTINI et al., 2013). Essas alterações provocam declínios na qualidade de vida e autonomia do idoso, sendo necessária ações para promoção de sua saúde.

Assim, devido à demanda da população local com relação às práticas de saúde na terceira idade, foi desenvolvido o Projeto de Extensão Vida Ativa. O projeto surgiu no ano de 2009, juntamente com o curso de Fisioterapia, da Universidade Federal de Alfenas (UNIFAL-

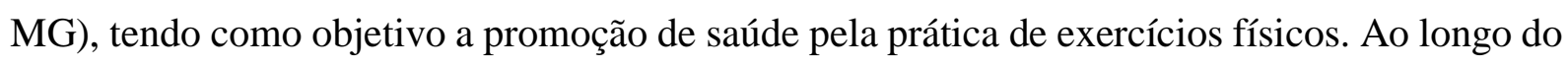
tempo, visto a necessidade da população local por mais ações de educação em saúde, foram incorporadas ao projeto ações interdisciplinares que atendessem aos idosos, contribuindo para formação de projeto de extensão que atende as cinco diretrizes propostas para ações de extensão: indissociabilidade, interdisciplinaridade, interação dialógica, impacto na formação do aluno e impacto social.

Devido à pandemia por COVID-19, houve a necessidade de distanciamento social como uma das principais medidas tomadas por governos ao redor do mundo com o objetivo de desacelerar o avanço da doença. Tal medida afetou a realização de diversas atividades desenvolvidas nas universidades e favoreceu a adaptação a plataformas online como alternativa para a continuidade do desenvolvimento de tais ações (AQUINO et al., 2020; SAHU, 2020).

Além da redução do nível de atividade física, o distanciamento social também causa efeitos psicológicos negativos nos indivíduos, sendo o medo de infecção, as perdas financeiras, informações falsas a respeito da doença, sentimentos de incerteza e aborrecimento alguns dos principais aspectos que afetam a saúde mental em períodos de quarentena (BARROS et al., 2020).

O contexto atual tornou necessário a adaptação do projeto, com o intuito de promover qualidade de vida, melhorar a capacidade funcional e independência para realizar atividades da vida diária, desenvolver atividades de ensino, pesquisa e extensão com acadêmicos do curso de Fisioterapia, desenvolver ações de educação em saúde interdisciplinar de forma acessível e

$$
\begin{array}{l|l}
\multirow{2}{*}{\text { REVISTA }} & \text { EXTENSÃO \& CIDADANIA } \\
\cline { 2 - 3 } & \text { v. 8, n. 14, p. 481-489, jul./dez. 2020. ISSN 2319-0566 }
\end{array}
$$


remota a comunidade idosa e promover inserção do idoso nas mídias sociais.

Baseado no exposto acima, o objetivo deste relato de experiência é expor as ações desenvolvidas pelos alunos e professores do Projeto Vida Ativa para pessoas idosas durante o período de isolamento social ocasionado pelo Covid-19.

\section{Metodologia}

Trata-se de estudo do tipo relato de experiência, que visa à descrição das ações e práticas desenvolvidas no ano de 2020, no contexto da pandemia pelo COVID-19, pelo projeto de extensão intitulado Vida Ativa inserido no Programa: Universidade Aberta à Terceira Idade, da Universidade Federal de Alfenas, que conta com a participação de duas docentes e 15 discentes do curso de Fisioterapia.

O projeto ocorre há 10 anos de forma presencial e, assim, após o período de férias retornou em fevereiro de 2020, sendo realizado avaliação motora, funcional e cognitiva dos participantes através dos testes: Timed $U p$ and Go e Medida de Independência Funcional (MIF). Diante da necessidade de isolamento como forma de contenção do avanço da pandemia, a partir de março de 2020 as atividades foram adaptadas para serem realizadas de forma remota. Atualmente, o projeto impacta cerca de 100 pessoas de forma remota pelo aplicativo WhatsApp ${ }^{\circledR}$, participam do projeto pessoas com idade igual ou superior a 60 anos.

Semanalmente, são enviados para os idosos vídeos com exercícios físicos seguros para a prática domiciliar, elaborados pelos alunos e professores participantes do projeto. Os vídeos são elaborados a partir das necessidades observadas pela ficha de avaliação realizada com os participantes antes do início do isolamento social e a partir de queixas que surgem no decorrer do projeto necessidade de exercícios para fortalecimento e alívio de dores na região de punho e mão, queixa relatada pelos participantes e por alunos, devido ao aumento da utilização de dispositivos eletrônicos nos últimos meses. Dessa forma, o projeto atua em diálogo com a sociedade, priorizando a troca de saberes pela interação dialógica.

Os vídeos e folders são produzidos pelos alunos participantes com o auxílio de

$$
\begin{array}{l|l}
\multirow{2}{*}{\text { REVISTA }} & \text { EXTENSÃO \& CIDADANIA } \\
\cline { 2 - 3 } & \text { v. 8, n. 14, p. 481-489, jul./dez. 2020. ISSN 2319-0566 }
\end{array}
$$


professores, elaborados pensando na segurança do idoso ao realizar as atividades sem auxílio presencial. Após o envio, os alunos se disponibilizam para sanar dúvidas que possam surgir sobre as atividades e em alguns casos são realizadas vídeo chamadas com os participantes para melhor compreensão.

A rede social Facebook® também é utilizada como plataforma para divulgação do projeto, sendo realizados posts semanais sobre temas diversos de educação em saúde e informações sobre o COVID-19, como uso correto de máscaras, lavagem das mãos, normas de segurança, importância de cumprir o isolamento social e os informativos relacionados à prevenção e controle de doenças crônicas, saúde mental no envelhecimento e prevenção do câncer de mama. Além disso, a página também é utilizada como forma de integração entre alunos, professores, idosos participantes e seus familiares.

São realizadas, pelos alunos com orientação de professores, de forma remota, discussões de temas explorados, a partir da necessidade dos idosos e discentes do projeto e apresentações de resultados de pesquisas de forma online em eventos científicos.

\section{Resultados}

Os idosos participantes diariamente relataram melhorias propiciadas pelas ações do projeto e como elas influenciaram positivamente a qualidade de vida durante o isolamento social. O uso das redes sociais contribuiu para redução da exclusão digital dos idosos e melhora da interação social. Além disso, a ação extensionista favoreceu a redução do sedentarismo comumente associado ao isolamento.

Para os acadêmicos participantes do projeto, a continuação da ação extensionista tem promovido maior envolvimento com os idosos, proporcionando interação dialógica, princípio básico da extensão, pelo desenvolvimento de atividades em conjunto com a comunidade.

A plataforma Facebook® alcançou 62 pessoas durante o período de isolamento, totalizando 319 curtidas até o momento, cerca de 260 pessoas são alcançadas semanalmente de forma orgânica de acordo com as análises e estatísticas do Facebook®. 
As apresentações de trabalhos científicos de forma online em congressos e simpósio, contribuem para a divulgação do conhecimento científico, promovendo a indissociabilidade da pesquisa, ensino e extensão, além de gerar impacto na formação do aluno, que experienciam vivências acadêmicas diversas em um único projeto.

\section{Discussão}

Pinheiro et al. (2020) destacam que o distanciamento social, apesar de essencial para os idosos, ocasiona diminuição no nível de atividade física praticado por esses indivíduos, o que propicia maior deterioração da saúde física, mental e facilita a instalação de doenças. Em contrapartida, sendo medida essencial para a minimização da exposição ao COVID-19, o isolamento social deixa os idosos desassistidos de medidas de promoção à saúde nesse período. Pouco tempo em confinamento e diminuição de atividade física já são suficientes para contribuir com a redução de força e massa muscular, equilíbrio e flexibilidade.

Segundo Girdhar, Srivastava e Sethi (2020), o isolamento social prolongado pode ser particularmente problemático para a saúde mental do idoso, pois está associado à constante preocupação com familiares, mudanças na dinâmica familiar, solidão, inatividade e diminuição da mobilidade. $\mathrm{O}$ incentivo à prática de exercícios físicos em domicílio pode ser usado como ferramenta no combate aos impactos negativos da quarentena na saúde física, mas também mental do idoso.

Jiménez-Pavón et al. (2020) mencionam a necessidade da prática de atividade física no período de isolamento e frente às mudanças drásticas de rotina, em função de manter o estado de saúde adequado, amenizando os efeitos deletérios de doenças como Diabetes Mellitus, hipertensão arterial sistêmica ou simplesmente com o objetivo de favorecer o envelhecimento saudável e ativo.

Visto a gama de problemas que podem afetar a qualidade de vida dos idosos em meio ao isolamento social, estratégias de enfrentamento do isolamento, como a do projeto Vida Ativa, necessitam de reconhecimento e apoio para sua continuação. É de extrema importância

$$
\begin{array}{l|l}
\multirow{2}{*}{\text { REVISTA }} & \text { EXTENSÃO \& CIDADANIA } \\
\cline { 2 - 3 } & \text { v. 8, n. 14, p. 481-489, jul./dez. 2020. ISSN 2319-0566 }
\end{array}
$$


viabilizar cada vez mais essas ações, tendo em vista os resultados que elas proporcionam aos idosos, familiares e aos serviços e instituições formativas que cercam essa população.

\section{Conclusão}

As práticas desenvolvidas pelo projeto durante pandemia possibilitaram a melhora da interação social dos idosos e da qualidade de vida. Com isso, o indivíduo tem sua autonomia resgatada pela prática de exercícios físicos e ações de educação em saúde, enquanto os acadêmicos experienciam a prática de saberes acadêmicos sendo inserida na comunidade. Em período de isolamento social, a estratégia adotada pelo projeto supracitado permite diálogo e interação com os idosos, reforçando a importância da atividade física para a saúde e a inclusão digital também de familiares e alunos.

O projeto proporciona formação cidadã aos alunos e interação com a comunidade. Assim, as atividades desenvolvidas têm repercussão positiva na manutenção e melhora da qualidade de vida dos idosos e impacto na formação do aluno, promovendo os princípios da extensão: indissociabilidade, interdisciplinaridade, interação dialógica, impacto na formação do aluno e impacto social. Portanto, ações de extensão em saúde devem ser estimuladas no ambiente universitário, mesmo que, de forma remota, pois contribuem para popularização do saber acadêmico na comunidade.

\section{Referências}

AQUINO, Estela M. L.; SILVEIRA, Ismael Henrique; PESCARINI, Julia Moreira; AQUINO, Rosana; SOUZA-FILHO, Jaime Almeida de; ROCHA, Aline dos Santos; FERREIRA, Andrea; VICTOR, Audêncio; TEIXEIRA, Camila; MACHADO, Daiane Borges. Medidas de distanciamento social no controle da pandemia de COVID-19: potenciais impactos e desafios no brasil. Ciência \& Saúde Coletiva, [S.L.], v. 25, n. 1, p. 2423-2446, jun. 2020. FapUNIFESP (SciELO). http://dx.doi.org/10.1590/1413-

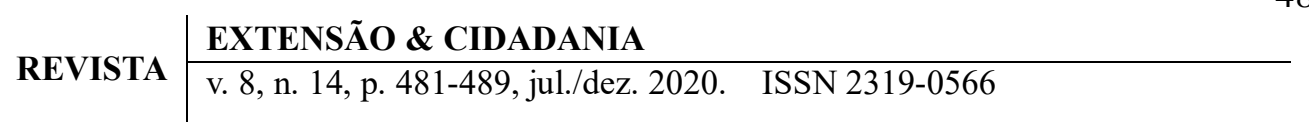


81232020256.1.10502020. Disponível em: https://www.scielo.br/scielo.php?pid=S1413$81232020006702423 \&$ script=sci_arttext. Acesso em: 10 out. 2020.

BARROS, Marilisa Berti de Azevedo et al. Relato de tristeza/depressão, nervosismo/ansiedade e problemas de sono na população adulta brasileira durante a pandemia de COVID-19. Epidemiologia e Serviços de Saúde, [S.L.], v. 29, n. 4, p. 1-12, 2020. FapUNIFESP (SciELO). http://dx.doi.org/10.1590/s1679-49742020000400018. Disponível em: https://www.scielo.br/scielo.php?script=sci_arttext\&pid=S223796222020000400311\&tlng=pt. Acesso em: 19 out. 2020.

CORTEZ, Antonio Carlos Leal; SILVA, Carlos Roberto Lyra; SILVA, Roberto Carlos Lyra; DANTAS, Estélio Henrique Martin. Aspectos gerais sobre a transição demográfica e epidemiológica da população brasileira. Enfermagem Brasil, [S.L.], v. 18, n. 5, p. 700-709, 8 nov. 2019. Atlantica Editora. http://dx.doi.org/10.33233/eb.v18i5.2785. Disponível em: http://portalatlanticaeditora.com.br/index.php/enfermagembrasil/article/view/2785. Acesso em: 19 out. 2020.

GIRDHAR, Ritika; SRIVASTAVA, Vivek; SETHI, Sujata. Managing mental health issues among elderly during COVID-19 pandemic. Journal of Geriatric Careand Research, v. 7, n. 1, p. 29-32, 2020.

Instituto Brasileiro de Geografia e Estatística. (IBGE) [INTERNET]. Projeções e estimativas da população do Brasil e das Unidades da Federação. Disponível em:

https://www.ibge.gov.br/apps/populacao/projecao/index.html?utm_source=portal\&utm_medi um=popclock. Acesso em: 10 jun. 2020.

JIMÉNEZ-PAVÓN, David et al. Physical exercise as therapy to fight against the mental and physical consequences of COVID-19 quarantine: special focus in older people. Progress in Cardiovascular Diseases, [S.L.], v. 63, n. 3, p. 386-388, maio 2020. Elsevier BV. http://dx.doi.org/10.1016/j.pcad.2020.03.009.

MELO, Anna Cássia Fernandes et al. Prevalência de doenças musculoesqueléticas autorreferidas segundo variáveis demográficas e de saúde: estudo transversal de idosos de Goiânia/GO. Caderno Saúde Coletiva. Rio de Janeiro, v. 25, n. 2, p. 138-143, abr. 2017. Disponível em: http://www.scielo.br/scielo.php?script=sci_arttext\&pid=S1414462X2017000200138\&lng=en\&nrm=iso. Acesso em: 27 maio 2020. https://doi.org/10.1590/1414-462x201700010274.

PASCOTINI, Fernanda dos Santos; RAMOS, Mônica de Castro; SILVA, Antônio Marcos Vargas da; TREVISAN, Maria Elaine. Espirometria de incentivo a volume versus a fluxo sobre parâmetros respiratórios em idosos. Fisioterapia e Pesquisa, [S.L.], v. 20, n. 4, p. 355 -

$$
\begin{array}{l|l}
\multirow{2}{*}{\text { REVISTA }} & \text { EXTENSÃO \& CIDADANIA } \\
\cline { 2 - 3 } & \text { v. 8, n. 14, p. 481-489, jul./dez. 2020. ISSN 2319-0566 }
\end{array}
$$


360, dez. 2013. FapUNIFESP (SciELO). http://dx.doi.org/10.1590/s1809-

29502013000400009. Disponível em:

https://www.scielo.br/scielo.php?script=sci_arttext\&pid=S1809-

29502013000400009\&lng=en\&nrm=iso. Acesso em: 20 set. 2020.

PINHEIRO, Jéssica da Silva et al. EXERCÍCIOS FÍSICOS NA SAÚDE DOS IDOSOS DURANTE A PANDEMIA POR COVID-19: uma revisão integrativa da literatura. Temas em Saúde - Edição Especial Covid, [S.L.], v. 1, n. 1, p. 25-38, 2020. Even3.

http://dx.doi.org/10.29327/224587.1.1-2. Disponível em: http://temasemsaude.com/wpcontent/uploads/2020/08/20covid2.pdf. Acesso em: 19 out. 2020.

SAHU, Pradeep. Closure of Universities Due to Coronavirus Disease 2019 (COVID-19): Impact on Education and Mental Health of Students and Academic Staff. Cureus Journal of Medical Science, [S. L.], v. 12, n. 4, p. 1-6, 12 abr. 2020. Disponível em:

https://www.ncbi.nlm.nih.gov/pmc/articles/PMC7198094/. Acesso em: 18 out. 2020.

Recebido em: 21 de outubro de 2020.

Aceito em: 8 de dezembro de 2020. 